\title{
Pigment epithelium-derived factor protects human glomerular mesangial cells from diabetes via NOXO1-iNOS suppression
}

\author{
LING GAO, YINGJIE HU and JING LI \\ Department of Endocrinology, Renmin Hospital of Wuhan University, Wuhan 430060, P.R. China
}

Received January 29, 2017; Accepted September 5, 2017

DOI: $10.3892 / \mathrm{mmr} .2017 .7563$

\begin{abstract}
The authors previously reported that pigment epithelium-derived factor (PEDF) protects the diabetic kidney from fibrosis via its anti-oxidative effects. However, the underlying molecular mechanism has never been revealed. The present study aimed to investigate how PEDF protects mesangial cells from diabetes induced damage. Human mesangial cells were exposed to a diabetic environment $[30 \mathrm{mmol} / 1$ glucose and $200 \mathrm{mg}$ advanced glycation end products (AGEs)] in the absence or presence of PEDF (200 mg/l). The superoxide and peroxynitrite productions were measured by fluorescent assay. The nicotinamide adenine dinucleotide phosphate (NAPDH) oxidases (NOXs; isoforms NOX1, NOX2, and NOX4), NADPH oxidase organizer 1 (NOXO1), DHFR, endothelial nitric oxide synthase (iNOS) and phospho-p38MAPK (p-p38) protein levels were also examined to explore the possible mechanism of the PEDF anti-oxidative properties. The fibrogenesis of mesangial cells in diabetes was associated with increased superoxide generation and peroxynitrite production via iNOS induction and uncoupling. However, elevated transforming growth factor- $\beta$ level, reactive oxygen species (ROS) overproduction, iNOS induction and uncoupling were all reversed by NOXO1 suppression following PEDF treatment or NOXO1 silencing. Furthermore, the p38MAPK inhibition only attenuated the ROS/peroxynitrite production partially via abolishment of iNOS induction, however had no effect on iNOS uncoupling and its regulating enzyme: DHFR suppression. PEDF prevented oxidative stress and protected mesangial cells from fibrogenesis in a diabetic environment via dual effects mediated by NOXO1 inhibitory prevention of iNOS induction through p38MAPK inactivation and effects on iNOS coupling through DHFR restoration.
\end{abstract}

Correspondence to: Professor Jing Li, Department of Endocrinology, Renmin Hospital of Wuhan University, 238 Jiefang Road, Wuhan, Hubei 430060, P.R. China

E-mail: lijing7823@medmail.com.cn

Key words: inducible nitric oxide synthase, human glomerular mesangial cells, tetrahydrobiopterin

\section{Introduction}

Diabetic nephropathy (DN) is a leading cause of end-stage renal failure worldwide. The pathogenetic mechanisms for DN may start with reactive oxygen species (ROS) as the common link connecting to various signaling pathways that lead to kidney impairment (1-4). The ROS are a family of molecules including superoxide anion $\left(\mathrm{O}^{2-}\right)$, hydroxyl racial $\left(\mathrm{HO}^{-}\right)$, hydrogen peroxide $\left(\mathrm{H}_{2} \mathrm{O}_{2}\right)$, peroxynitrite $\left(\mathrm{ONOO}^{-}\right)$, nitric oxide (NO) and lipid radicals. In mammalian cells, potential origins of ROS generation include mitochondrial respiratory chain, xanthine oxidase, NOXs, and nitric oxide synthase (NOS), with increasing evidence that NOXs appear to be the major sources or more specifically initial trigger of ROS generation in the diabetic complications including kidney (5-7).

Various subtypes of NOXs (e.g., NOX1, NOX2, NOX4) are discovered abundantly in the mesangial cells (8). Lee et al (9)showed that mitochondrial derived ROS was elevated in DN subsequent to NOX4 activation. Although NOX4 is likely implicated in the mediation of kidney fibrosis in diabetes including mesangial impairment, other subtypes such as NOX1, NOX2 and their cytosolic component NADPH oxidase organizer 1 (NOXO1) are also possible candidates involved in this pathogenesis (10). Additionally, other researches reported that NOS uncoupling instead of mitochondria dysfunction in the mesangial cells is responsible for the early glomerular pathology in diabetes and is associated with inflammation and oxidative stress of DN (11). Thus, the downstream of NOXs activation or the main source of oxidative stress is still in debate and should be under extensive investigation. Recently, both in vivo and in vitro studies have demonstrated that nitroxidative/nitrosative stress (ONOO-) has been actively involved in the development of DN or the hyperglycemia induced damage in mesangial cells (12). Therefore, NOS uncoupling as the most possible cause of $\mathrm{ONOO}^{-}$production should be examined. It is well known that NOS coupling status is governed by its cofactor tetrahydrobiopterin (H4B) abundance, for instance: when H4B is deficient, NOS becomes dysfunctional to produce superoxide instead of $\mathrm{NO}$ which changes into $\mathrm{ONOO}^{-}$on site instantly and vastly. Moreover, H4B synethesis is controlled by dihydrofolate reductase (DHFR), the key enzyme responsible for H4B salvage pathway, whose expression level would determine the abundance of H4B. Notably, DHFR suppression 
could play an important role in the development of DN via dysfunctional NOS and restoration of DHFR may well be the target for antioxidant treatment.

As an antioxidant, epithelium-derived factor (PEDF) is a glycoprotein that belongs to the superfamily of serine protease inhibitors, which is assumed to have beneficial effects on retinopathy. It was first retrieved from the culture media of human retinal pigment epithelial cells which possessed potential neuronal differentiating activity. PEDF is expressed broadly in human tissues, including the kidney. Previous studies have shown that decreased PEDF levels in the kidney are implicated in DN, especially in the early stage (13). It is reported that the injection of adenovirus expressing PEDF recombinant gene significantly reduced the albuminuria and the production of extracellular matrix (ECM) protein, and ameliorated glomerular hypertrophy in the diabetic kidney (14). Although, according to previous studies, it is assumed that PEDF protects the renal function from diabetic injury, via its anti-oxidative and anti-fibrogenic activities, the particular mechanism has not been fully illustrated (15-18). Therefore, the purpose of the present study is to investigate how PEDF protects the kidney from oxidative stress and determine whether this beneficiary effect is through rectifying dysfunctional iNOS and de-activation of NOXs including NOXO1.

\section{Materials and methods}

Cell culture. The use of human glomerular mesangial cells (HMCs; Xiangya School of Medicine, Central South University, Changsha, China) in the present study was approved by the Ethics Committee of Renmin Hospital of Wuhan University (Wuhan, China) which was in adherence with the Declaration of Helsinky. Dulbecco Modified Eagle's Medium (DMEM) were used to culture HMCs. Cells of passages 3-6 were used and after reaching nearly confluence, they were quiescenced with serum starvation for $48 \mathrm{~h}$, and then exposed to different conditions without elevated glucose or advanced glycation end products (AGEs) in the presence or absence of PEDF for $48 \mathrm{~h}$. In some experiments, the cells were also treated with $20 \mu \mathrm{M} \mathrm{SB} 203580$ for p38MAPK inhibition. Bovine serum albumin (BSA; $200 \mathrm{mg} / \mathrm{l}$ ) was used as a control for AGE treatment. The PEDF was purchased from Peprotech (Princeton, USA); AGEs were purchased from Jiamay Biotech (Beijing, China); the rest mediums and reagents were purchased from Sigma-Aldrich (Merck KGaA; Darmstadt, Germany) unless stated otherwise. PEDF at $100 \mathrm{nmol} / 1$ was used in this study since our previous dose-dependent experiment showed that this was a proper dose for the antioxidant protection of mesangial cells (18).

Western blotting analysis. The protein levels of NOX1, NOXO1, NOX2, NOX4, DHFR, iNOS and p-p38MAPK were detected by immunoblotting analysis. After the treatment, cells were lysed in $50 \mathrm{mM}$ Tris-HCl buffer. Equal amounts of lysates $(20-40 \mu \mathrm{g})$ were loaded, separated by $10 \%$ SDS-PAGE and transferred to PVDF membranes. Membranes were then blocked with 5\% milk overnight, and blotted with primary antibody against TGF- $\beta$ (Rabbit; 1:500); iNOS (mouse; 1:1,000); NOX1 (Goat; 1:1,000), NOXO1 (Rabbit; 1:1,000);
NOX2 (Rabbit; 1:1,000) and NOX4 (Rabbit; 1:1,000), DHFR (Rabbit; 1:1,000; all Abcam, Cambridge, UK) and p-p38MAPK (Rabbit; 1:1,000; Cell Signaling Technology, Inc., Danvers, MA, USA) separately following standard procedure. The protein band density was measured by Image $\mathrm{J}$ software and the quantification was reflective of the relative amounts as a ratio of each protein band over loading control ( $\beta$-actin).

Measurements of TGF- $\beta 1$ by enzyme-linked immunosorbent assay (ELISA). Spectrophotometre was used to detect the protein levels of TGF- $\beta 1$ and FN which was prepared with the FN ELISA kit (USCN Life Sciences, Inc., Wuhan, China) and the TGF- $\beta 1$ ELISA kit (Boster, Wuhan, China) according to the manufacturers' protocol.

In vitro silence of NOXO1. The RNAi against NOXO1 (5'-CCTCGCCCATTTCAGGAAT-3') was obtained from Invitrogen (Thermo Fisher Scientific Inc., Waltham, MA, USA). HMCs were transfected with siRNA/oligofectamine (Invitrogen; Thermo Fisher Scientific Inc.) mixtures according to the manufacturer's protocol.

HPLC-based $\mathrm{H}_{4} B$ measurement. HMCs were lysed by trichloroacetic acid containing $10 \mathrm{mmol} / \mathrm{l}$ DTT. Lysates were subjected to differential oxidation in acidic or alkalytic solutions (19). In summary, total biopterin (H4B, dihydropterin [H2B], and oxidized biopterin) were determined by acid oxidation, whereas $\mathrm{H} 2 \mathrm{~B}$ and oxidized biopterin were measured by alkali oxidation. The ratio of H4B over H2B was calculated and used as an index for DHFR activity.

Intracellular $\mathrm{ONOO}^{-}$measurement. $\mathrm{ONOO}^{-}$contents were detected using hydroxyphenyl fluorescein (HPF) (20). Briefly, cell suspension $\left(200 \mu \mathrm{l}, 10^{7} / \mathrm{ml}\right)$ was incubated with $10 \mu \mathrm{M}$ $\mathrm{HPF}$ in 96 well plates for $30 \mathrm{~min}$ at $37^{\circ} \mathrm{C}$ in the dark. The fluorescence generated was measured at excitation $485 \mathrm{~nm}$ and emission $585 \mathrm{~nm}$.

Statistical analysis. One-way ANOVA was used to test for statistical significance, and $\mathrm{P}<0.05$ was considered to indicate a statistically significant difference. All data shown in the figures were presented as means $\pm \mathrm{SD}$.

\section{Results}

PEDF abolishes iNOS upregulation induced by diabetic environment via p38MAPK inhibition. Both $\mathrm{p} 38 \mathrm{MAPK}$ and iNOS protein levels were upregulated in diabetic environment (Fig. 1). However, p38MAPK and iNOS upregulations were annihilated by PEDF treatment (Fig. 1A-C). Moreover, p38MAPK inhibitor, SB203580 abolished iNOS induction completely but only canceled $\mathrm{ONOO}^{-}$and TGF- $\beta$ overproduction partially (Fig. 1E-H). In comparison, iNOS induction, peroxynitite and TGF- $\beta$ overproduction were resumed back to the normal level by PEDF (Fig. 1A-H).

PEDF reverses $i N O S$ uncoupling induced by diabetic environment. H4B is the cofactor for iNOS and its abundance decides the coupling status of iNOS. DHFR is a key enzyme of H4B 

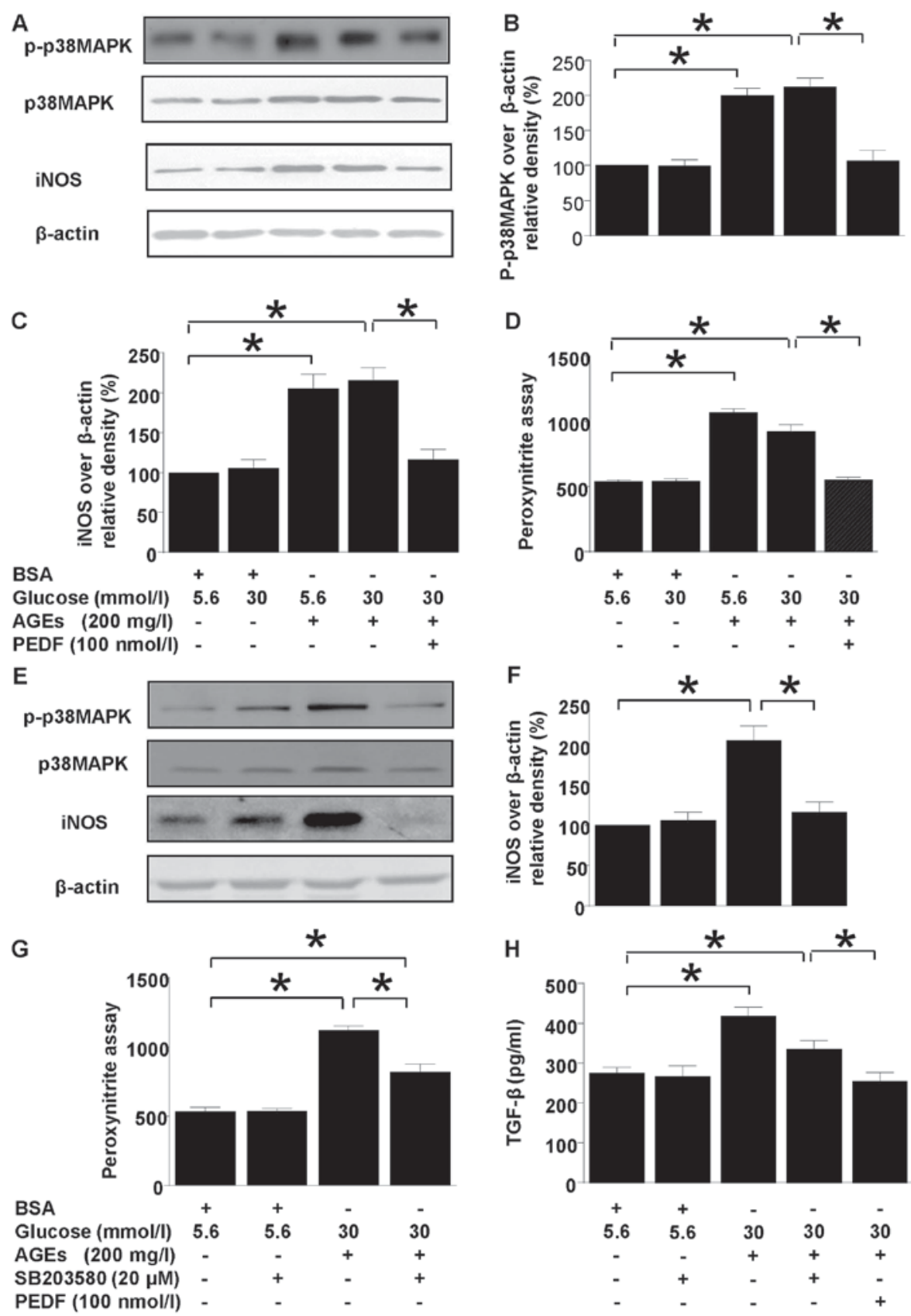

Figure 1. Effects of PEDF or p38MAPK inhibition on iNOS and peroxynitrite production. Cells were cultured in $5.6 \mathrm{mmol} / 1 \mathrm{glucose}, 30 \mathrm{mmol} / \mathrm{lglucose}$, $5.6 \mathrm{mmol} / 1$ glucose $+200 \mathrm{mg} / \mathrm{l} \mathrm{AGEs,} 30 \mathrm{mmol} / 1$ glucose $+200 \mathrm{mg} / \mathrm{l}$ AGEs and $30 \mathrm{mmol} / 1 \mathrm{glucose}+200 \mathrm{mg} / \mathrm{l} \mathrm{AGEs}+100 \mathrm{nmol} / 1 \mathrm{PEDF}$ for $48 \mathrm{~h}$ (A-D) or cells were cultured in $5.6 \mathrm{mmol} / 1$ glucose, $5.6 \mathrm{mmol} / 1$ glucose $+20 \mu \mathrm{mol} / 1 \mathrm{SB} 203580,30 \mathrm{mmol} / 1$ glucose $+200 \mathrm{mg} / 1 \mathrm{AGEs}$, and $30 \mathrm{mmol} / 1 \mathrm{glucose}+200 \mathrm{mg} / \mathrm{l}$ AGEs $+20 \mu \mathrm{mol} / 1 \mathrm{SB} 203580$ for $48 \mathrm{~h}$ (E-H). (A) p-p38MAPK, iNOS and $\beta$-actin protein levels were assessed by immunoblotting analysis; (B) Relative p-p38 MAPK blot density was analyzed and plotted; (C and F) The density analysis of iNOS/ $\beta$-actin from immunoblots; (D and G) The peroxynitrite production was analyzed by HPF fluorescence assay; (E) p-p38MAPK, iNOS and $\beta$-actin protein levels were assessed by immunoblotting analysis in the presence or absence of SB 203580. (H) The TGF- $\beta$ expression tested by ELISA. Data are expressed as the means \pm standard error of the mean, $n=3-5$ and ${ }^{*} \mathrm{P}<0.05$ as indicated. PEDF, pigment epithelium-derived factor; iNOS, nitric oxide synthase; AGEs, advanced glycation end products; TGF, transforming growth factor; BSA, bovine serum albumin; MAPK, mitogen activated protein kinase; p, phosphorylated.

salvage pathway which could modulate iNOS coupling status via regulation of H4B synthesis (Fig. 2). Interestingly, DHFR protein level was downregulated in diabetic environment and PEDF retrieved DHFR protein level back to basal level (Fig. 2A and $\mathrm{C}$ ). As a result of DHFR reduction in diabetes environment, the $\mathrm{H} 4 \mathrm{~B}$ contents and $\mathrm{H} 4 \mathrm{~B} / \mathrm{H} 2 \mathrm{~B}$ ratio were diminished (Fig. 2E-H), but PEDF restored H4B level as well as $\mathrm{H} 4 \mathrm{~B} / \mathrm{H} 2 \mathrm{~B}$ ratio in accordance with upregulation of DHFR protein level (Fig. 2E, G). However, SB203580 had no effect on
DHFR protein expression, H4B contents as well as H4B/H2B ratio (Fig. 2B, D, F and $\mathrm{H}$ ).

PEDF acts independent of NOXs. Our previous study has shown that NOX1-iNOS pathway played a major role in diabetes induced oxidative stress of mesangial cells. However, this study showed that although NOX1, NOX2 and NOX4 were all upregulated in diabetes environment, PEDF had no effect on their protein levels (Fig. 3), suggesting that 
A

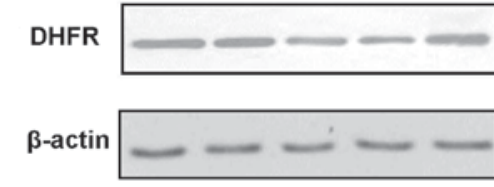

C

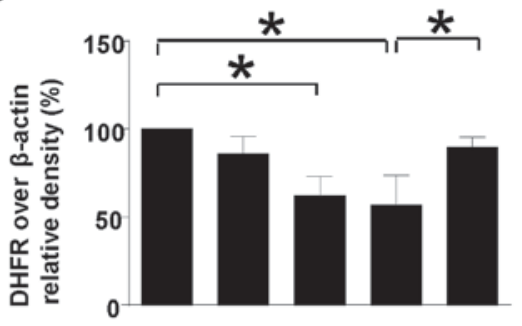

E
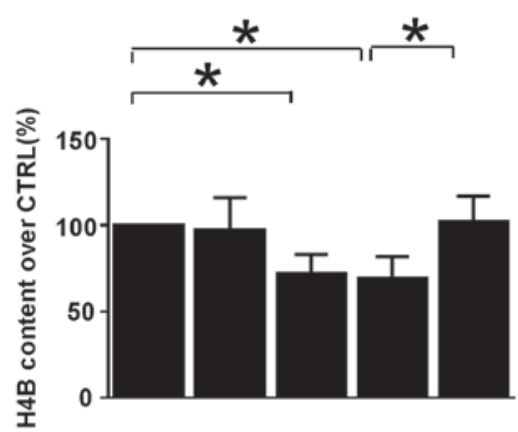

G

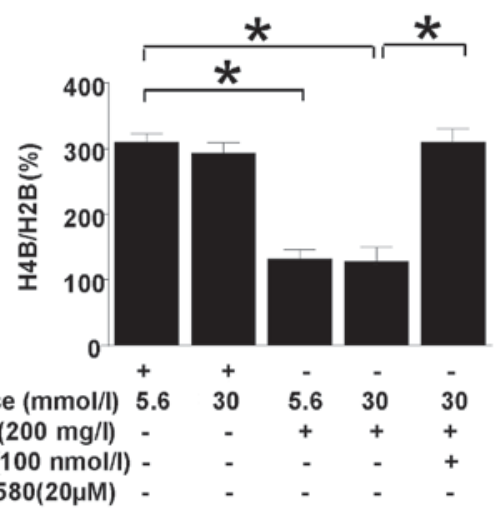

B
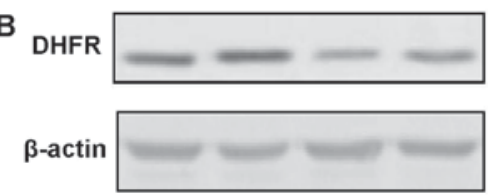

D

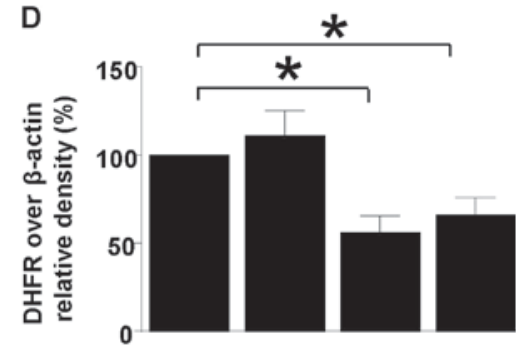

$\mathbf{F}$

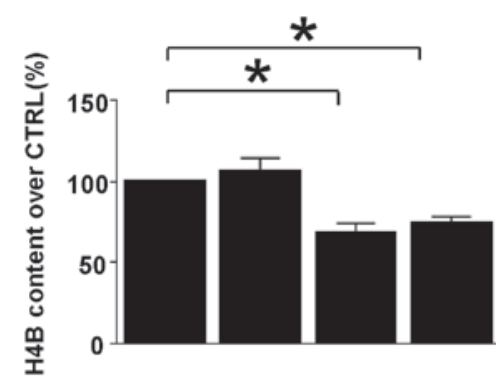

H
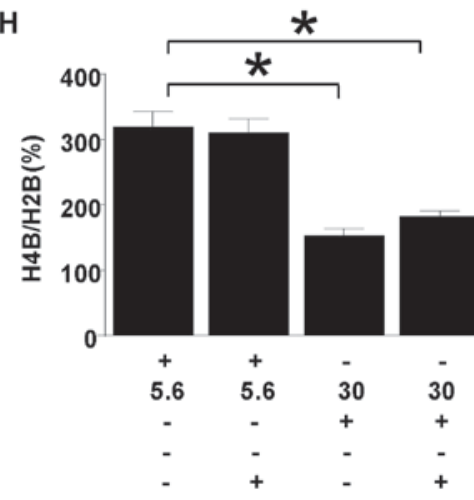

Figure 2. The effects of PEDF or SB203580 on DHFR protein expression and intracellular H4B contents. Cells were cultured in $5.6 \mathrm{mmol} / \mathrm{l}$ glucose, $30 \mathrm{mmol} / 1$ glucose, $5.6 \mathrm{mmol} / 1$ glucose $+200 \mathrm{mg} / 1$ AGEs, $30 \mathrm{mmol} / 1$ glucose $+200 \mathrm{mg} / 1$ AGEs and $30 \mathrm{mmol} / 1 \mathrm{glucose}+200 \mathrm{mg} / 1 \mathrm{AGEs}+100 \mathrm{nmol} / 1$ PEDF for $48 \mathrm{~h}$ (A, C, E), or cells were cultured in $5.6 \mathrm{mmol} / 1$ glucose, $5.6 \mathrm{mmol} / 1$ glucose $+20 \mu \mathrm{mol} / 1 \mathrm{SB} 203580,30 \mathrm{mmol} / 1 \mathrm{glucose}+200 \mathrm{mg} / 1 \mathrm{AGEs}$ and $30 \mathrm{mmol} / 1$ glucose $+200 \mathrm{mg} / \mathrm{l}$ AGEs $+20 \mu \mathrm{mol} / 1 \mathrm{SB} 203580$ for $48 \mathrm{~h}$ (B, D, F). (A and B) DHFR and $\beta$-actin protein levels were assessed by immunoblotting analysis; (C and D) The density analysis of DHFR/ $\beta$-actin from immunoblot; ( $E$ and F) the intracellular H4B contents were measured with high-performance liquid chromatography; $(\mathrm{G}$ and $\mathrm{H})$ the ratio of $\mathrm{H} 4 \mathrm{~B} / \mathrm{H} 2 \mathrm{~B}$ was calculated and analyzed. Data are expressed as the means \pm standard error of the mean, $\mathrm{n}=3-5$ and " $\mathrm{P}<0.05$ as indicated. PEDF, pigment epithelium-derived factor; AGEs, advanced glycation end products; H4B, tetrahydrobiopterin; H2B, dihydropterin; CTRL, control; DHFR, dihydrofolate reductase; BSA, bovine serum albumin.

PEDF may protect kidney at probably the downstream of NOX1 pathway. However, NOXO1, the adaptor protein for NOX1 was downregulated by PEDF suggesting that NOXO1 suppression could be responsible for the protective mechanism of PEDF.

PEDF confers renal protection via NOXOI inhibition. Silencing NOXO1 with RNAi downregulated NOXO1 protein expression (Fig. 4) and reproduced the effect of PEDF on iNOS expression and coupling status. NOXO1 silencing abolished the iNOS suppression (Fig. 4A and C). Meanwhile, uncoupling of iNOS was also annihilated by NOXO1 silencing as demonstrated by measurement of DHFR expression (Fig. 4D), H4B contents, and H4B/H2B ratio (Fig. 4D and E). Consequently, in mesangial cells the protective effect of PEDF on oxidative stress (ONOO- overproduction, Fig. 4G) and kidney fibrosis (TFGF- $\beta$ production $4 \mathrm{H}$ ) were also duplicated by NOXO1 silencing.

\section{Discussion}

The present study explored the possible mechanism or the molecular pathway of PEDF action on mesangial cells in diabetes. We found that PEDF was protective for mesangial cells in diabetic environment by reversing TGF- $\beta$ overexpression, $\mathrm{ONOO}^{-}$overproduction, iNOS induction and uncoupling. 
A

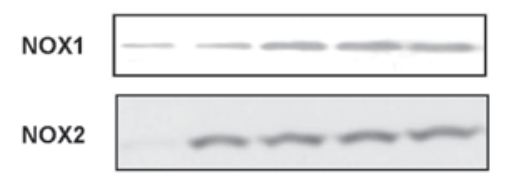

NOX4

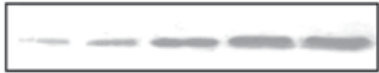

NOX01

$\beta$-actin
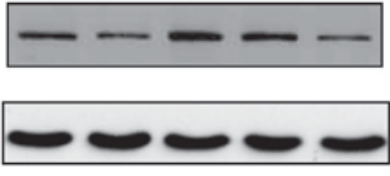

C
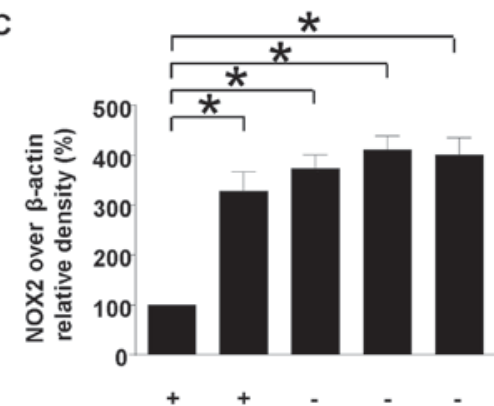

$\begin{array}{llllll}\text { BSA } & + & + & - & - & - \\ \text { Glucose }(\mathrm{mmol} / \mathrm{l}) & 5.6 & 30 & 5.6 & 30 & 30\end{array}$

AGEs $(200 \mathrm{mg} / \mathrm{l})-\quad-\quad+\quad+$

PEDF (100 nmol/l) - $\quad-\quad$ - $\quad-\quad+$

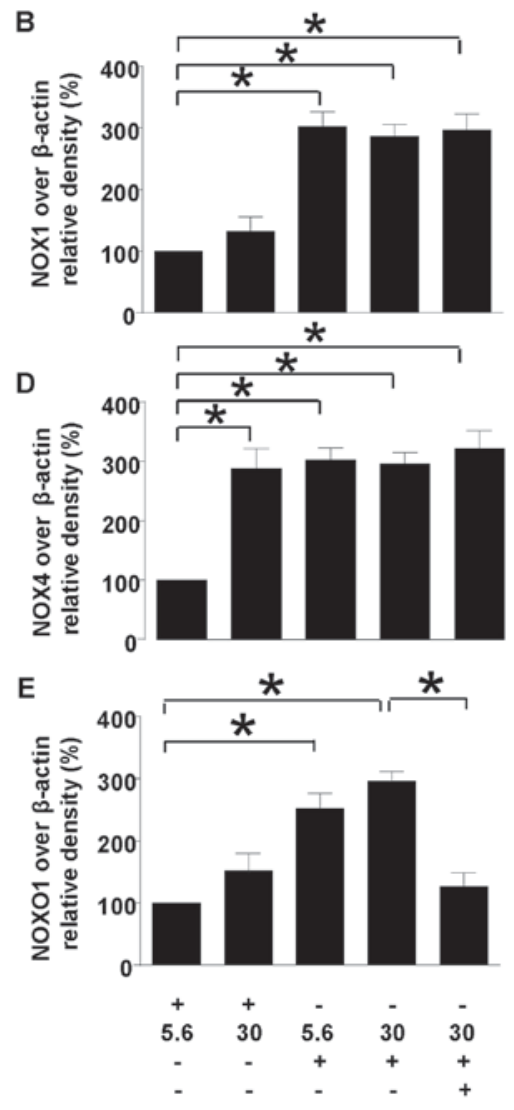

B

D

Figure 3. Effects of PEDF on NOXs activation. Cells were cultured in $5.6 \mathrm{mmol} / 1 \mathrm{glucose}, 30 \mathrm{mmol} / 1 \mathrm{glucose}, 5.6 \mathrm{mmol} / 1 \mathrm{glucose}+200 \mathrm{mg} / 1 \mathrm{AGEs}$, $30 \mathrm{mmol} / 1$ glucose $+200 \mathrm{mg} / 1$ AGEs and $30 \mathrm{mmol} / 1$ glucose $+200 \mathrm{mg} / 1$ AGEs+100 nmol/1 PEDF for 48 h. (A) NOX1, NOX2, NOX4, NOXO1 and $\beta$-actin protein levels were assessed by immunoblotting analysis; (B) The density analysis of NOX2/ $\beta$-actin from immunoblot; (C) The density analysis of NOX4/ $\beta$-actin from immunoblot; (D) The density analysis of NOX1/ $\beta$-actin from immunoblot; (E) The density analysis of NOXO1/ $\beta$-actin from immunoblot; Data are expressed as the means \pm standard error of the mean, $\mathrm{n}=3-5$ and ${ }^{*} \mathrm{P}<0.05$ as indicated. PEDF, pigment epithelium-derived factor; NOXs, nicotinamide adenine dinucleotide phosphate (NAPDH) oxidases; BSA, bovine serum albumin.

The ONOO- derived from iNOS induction and uncoupling was completely abolished consequent to PEDF treatment via $\mathrm{p} 38 \mathrm{MAPK}$ inactivation and restoration of DHFR protein level. However, those salutary effects of PEDF were mediated by NOXO1 but not individual NOXs downregulation. Taken together, our findings provided initial evidence to reveal novel mechanisms that PEDF prevented oxidative stress and protected mesangial cells from fibrogenesis in diabetic environment via dual effects converging at NOXO1 suppression: Retrivation of iNOS overexpression through p38MAPK inactivation and restoration of iNOS coupling through DHFR restoration.

Sheikpranbabu et al (21) have shown that PEDF significantly decreased NADPH oxidase and ROS generation in pericytes following AGE-BSA treatment. In the present study, we observed an attenuation of ROS production by PEDF in mesangial cells treated with high glucose and AGEs (diabetes environment). As NOX1, NOX2 and NOX4 are the three major NADPH oxidases existed in the mesangial cells and our previous study showed that the activation of NOX1 and its derived superoxide led to direct cell injury and initiated a chain of deleterious stress signaling such as iNOS induction and uncoupling. The effect of PEDF on NOXs protein level was examined (22). However, NOXs protein level was not altered by PEDF. Interestingly, NOXO1, the cytosolic regulation protein for NOXs (23), was suppressed by PEDF and downregulation of NOXO1 by RNA silencing reproduced the antioxidant effect of PEDF and its restoration effect on iNOS induction and uncoupling. All of these suggested that NOXO1 mediated the PEDF protective effect.

It has been demonstrated that p38MAPK activation is involved in the development of DN (24). However, the exact role of p38MAPK is not clear since it is traditionally considered as an anti-apoptotic signaling molecule. The p38MAPK activation is not self explanatory for the mesangial cells proliferation in the early stage of DN. Our data, among a few other researches suggested that p38MAPK is a pro-fibrogenetic rather than a pro-apoptotic molecule in high glucose induced mesangial damage $(25,26)$.

Previously, it was suggested that p38MAPK was a possible upstream for iNOS and it has been reported that p38MAPK is activated in glomeruli isolated from streptozotocin-induced diabetic rats and glomerular mesangial cells cultured under high glucose conditions (27). Yuan et al (28) showed that high glucose $(33 \mathrm{mM})$ caused activation of p38MAPK and inhibition for p38MAPK abrogated the high glucose induced iNOS expression, cell injury and levels of $\mathrm{NO}$ and nitrotyrosine in cultured human retinal pigmented epithelial cells. Therefore, activation of p38MAPK plays an important role in the iNOS induction and oxidative stress of DN. Recently, ATF-2 and NF-KB are suggested to be the possible downstreams or targets of p38MAPK whose activation may contribute to iNOS 
A
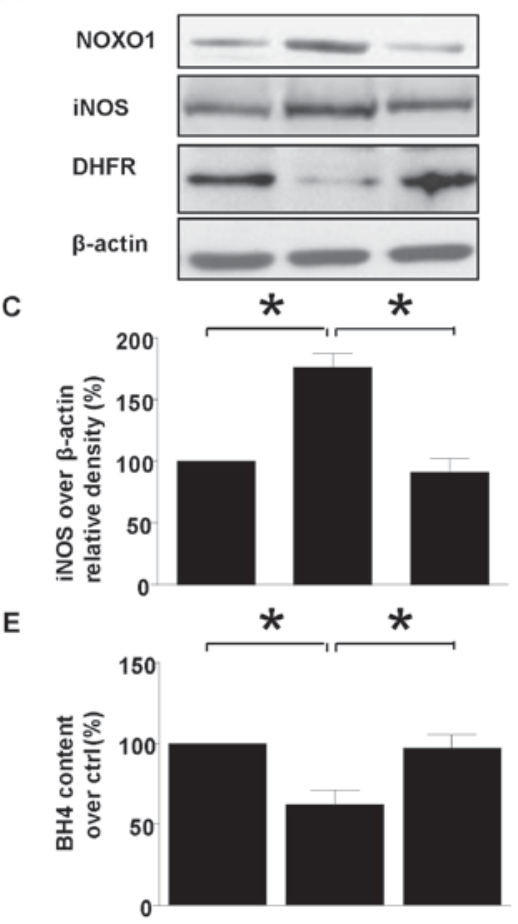

G

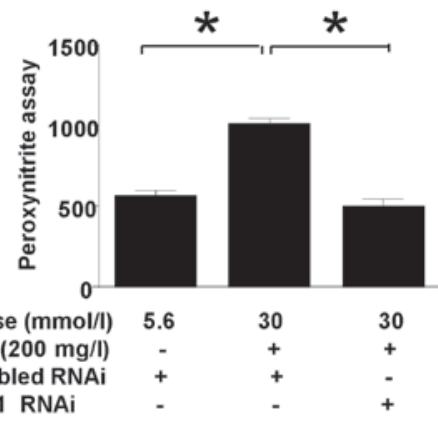

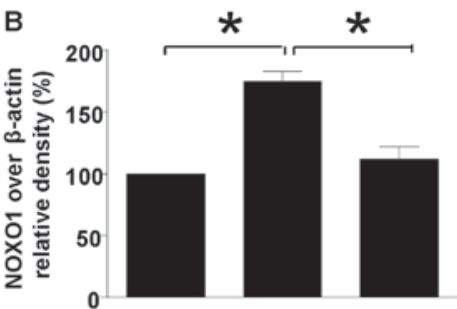

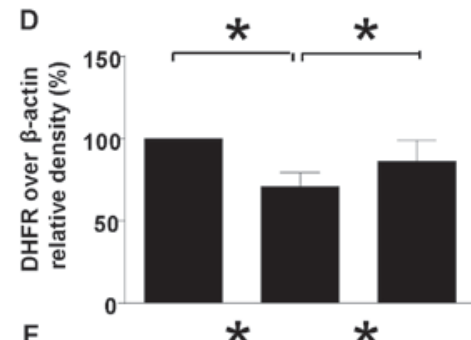

$\mathbf{F}$
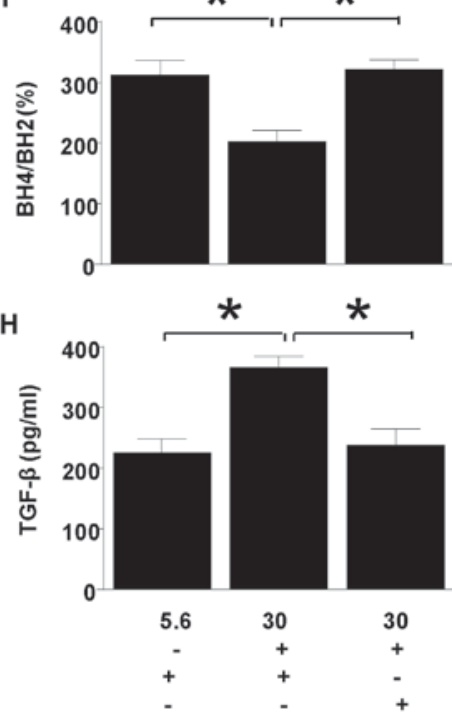

Figure 4. The effect of NOXO1 silencing on diabetes induced iNOS upregulation and uncoupling. Cells were cultured in $5.6 \mathrm{mmol} / 1 \mathrm{glucose}$, $30 \mathrm{mmol} / 1$ glucose $+200 \mathrm{mg} / 1$ AGEs in the presence or absence of scrambled RNAi or NOXO1 RNAi for $48 \mathrm{~h}$. (A) NOXO1, DHFR, iNOS and $\beta$-actin protein levels were assessed by immunoblotting; (B) The density analysis of NOXO1/ $\beta$-actin from immunoblot; (C) The density analysis of iNOS/ $\beta$-actin from immunoblot; (D) The density analysis of DHFR/ $\beta$-actin from immunoblot. (E) Intracellular H4B content was measured by high-performance liquid chromatography; (F) The ratio of $\mathrm{H} 4 \mathrm{~B} / \mathrm{H} 2 \mathrm{~B}$ was calculated and analyzed; (G) Peroxynitrite (ONOO) production was examined by hydroxyphenyl fluorescein staining; (H) TGF- $\beta$ protein levels were measured using ELISA. Data are expressed as the means \pm standard error of the mean, $n=3-5$ and ${ }^{*} \mathrm{P}<0.05$ as indicated. iNOS, inducible nitric oxide synthase; NOXO1, nicotinamide adenine dinucleotide phosphate oxidase 1; AGEs, advanced glycation end products; H4B, tetrahydrobiopterin; H2B, dihydropterin; DHFR, dihydrofolate reductase; RNAi, RNA inteference; TGF, transforming growth factor.

unregulation (29). In this study, we established that iNOS was induced in diabetes environment consequent to p38MAPK upregulation, but PEDF reversed the process. However, the p38MAPK inhibitor, SB203580 completely abolished iNOS induction but only partially canceled $\mathrm{ONOO}^{-}$and TGF- $\beta$ overproduction in diabetic enviroment. Therefore, we speculated that PEDF may protect kidney from diabetes via multiple pathways including p38MAPK and iNOS induction.

In the endothelial cells, PEDF has been shown to inhibit the cellular permeability via p38MAPK de-activation (30). While, PEDF also protected against retinal neovascularization by suppression of p38MAPK activation and dampening iNOS induction in the macrophage $(31,32)$. Likewise, our data showed that PEDF, by targeting NOXO1, protected mesangial cells from fibrogenesis and nitroxidative/nitrosative stress, at least partly via abrogation of p38MAPK mediated iNOS induction.
It is well established that oxidative stress has a major role in the development of diabetic complications including DN. ONOO-, as a notorious radical form created from the interaction between superoxide and NO, attacks various biomolecules in the tissues and induces damages in the microcirculations $(33,34)$. Prabhakar et al (12) reported that renal $\mathrm{ONOO}^{-}$formation, which acts as a main contributor to oxidative stress of DN and accounts partly for decreased NO bioavailability of kidney, was elevated in the kidney homogenates of obese ZSF1 rats ( $\sim 150 \%$ up) vs. lean ZSF rats. However, there is a lack of quantitative information of $\mathrm{ONOO}^{-}$about the formation and biological relevance because of its difficult and indirect measurements. Indeed, serum level of oxidative stress indicated by $\mathrm{ONOO}^{-}$is associated with the severity of diseases such as Parkinson's (35).

Since iNOS is the main source for $\mathrm{ONOO}^{-}$overproduction, iNOS uncoupling could be another contributing factor for the 


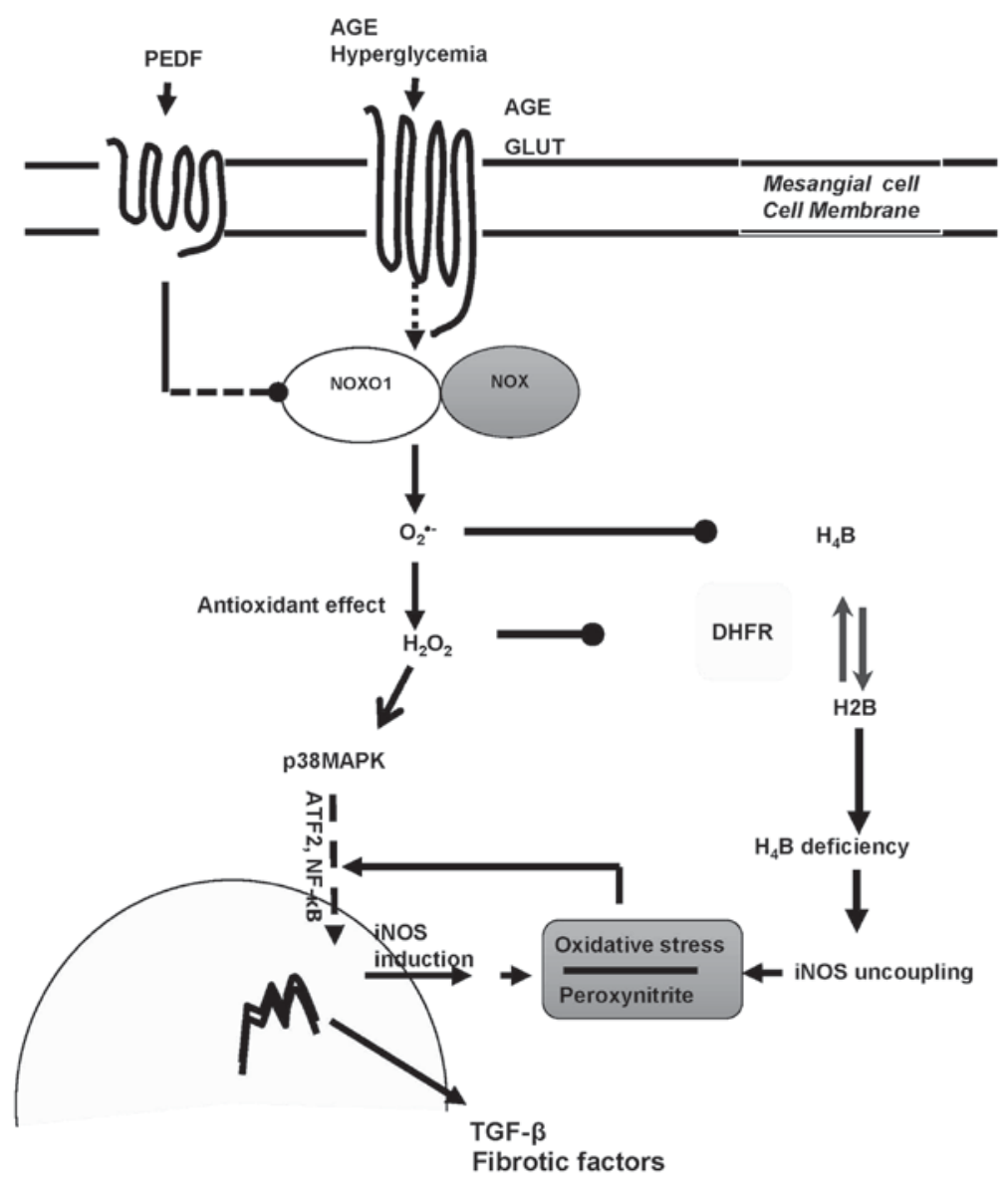

Figure 5. The dual mechanisms of PEDF on diabetic kidney protection. PEDF prevented oxidative stress and protected against fibrogenesis of mesangial cells in diabetic environment via dual effects mediated by NOXO1 inhibition: Annhilation of iNOS induction through p38MAPK inactivation and retrivation of iNOS coupling through DHFR restoration. PEDF, pigment epithelium-derived factor; iNOS, inducible nitric oxide synthase; NOXO1, nicotinamide adenine dinucleotide phosphate oxidase 1; AGE, advanced glycation end product; H4B, tetrahydrobiopterin; H2B, dihydropterin; DHFR, dihydrofolate reductase; TGF, transforming growth factor; NF, nuclear factor; GLUT, glucose transporter; MAPK, mitogen activated protein kinase; ATF. Activating transcription factor.

nitrosative stress. Basically, when iNOS becomes uncoupled, it will fail to produce NO, and begin to produce superoxide which eventually culminates in $\mathrm{ONOO}^{-}$formation. $\mathrm{H} 4 \mathrm{~B}$ is the key co-factor for iNOS whose deficiency results in iNOS uncoupling. Indeed, DHFR (the enzyme responsible for H4B replenishment) is diminished in diabetes environment which consequently leads to H4B consumption (36). As we expected, PEDF relieved the suppression of DHFR and retrieved H4B contents $(\mathrm{H} 4 \mathrm{~B} / \mathrm{H} 2 \mathrm{~B}$ ratio) in diabetes which eventually lead to iNOS recoupling. This effect is independent of p38MAPK pathway because SB203508, the p38MAPK inhibitor has no effect on DHFR suppression and H4B deficiency.

The deleterious effects of diabetes are attributed mainly to the formation of sugar-derived substances called AGEs. The AGEs formation is markedly accelerated in diabetes because of the increased availability of glucose. We always believe that the detrimental effects of diabetes are the combination of AGEs and elevated glucose, although $\mathrm{ONOO}^{-}$formation is majorly due to AGEs, not elevated glucose. Likewise, the AGE effect is more detrimental and advanced damage. On the other hand, the effect of high glucose is more of an acute damage and represents an early stage of oxidative stress such as NOX activation (37,38). Overall, AGEs represented the chronic effect of diabetic condition on renal impairment, and elevated glucose acted as an acute insult (within min). In our study, the cells were examined after $48 \mathrm{~h}$ during which the acute effect of elevated glucose may have faded away (so as the glucose concentration) and its extended effect may act via protein glycation which is AGEs-related. Nevertheless, it is important to expose the mesangial cells with elevated glucose and AGE together in order to mimic the in vivo diabetic condition.

NOXO1 is originally believed to be the cytosolic or inner component/subunit that solely regulates NOX1 activity/mediated superoxide generation, but is also recently found to interact with other NOXs such as NOX2, NOX4 and be required for their assembly and activation. Collectively, it is still unclear about NOXO1 regarding its function in the mesangial cells. The current data suggests that although NOX1, NOX2, NOX4 and NOXO1 are involved in the high glucose induced oxidative stress in the mesangial cells, only NOXO1 has a key role in the regulation of detrimental events. Moreover, since NOX1, NOX2 and NOX4 all possibly interact with NOXO1 $(10,39)$, they could be linked to the effect of PEDF as a complex with NOXO1.

In summary, iNOS is the main source of ROS in diabetic kidney and PEDF protects against diabetic damage via iNOS suppression mediated by $\mathrm{p} 38 \mathrm{MAPK}$ inactivation and iNOS recoupling via H4B restoration consequent to DHFR upregulation (Fig. 5). Therefore, we have verified the antioxidant effects of PEDF, which is consistent with previous studies. This 
is the first time we clarify the anti-oxidation mechanism of PEDF on DN which could lead to the discovery of a potential target for PEDF and provide a theoretical basis for the clinical treatment of DN. It is noticed in this study that PEDF curbed the origination and progression of oxidative stress by NOXO1 suppression, but the mechanism of PEDF on the initial link of oxidative stress is still puzzled.

\section{Acknowledgements}

The present study is supported by National Natural Science Foundation of China (grant nos. 81170767 and 81571376 to Dr Gao) and Diabetes Study Fund from Chinese Medical Association (grant no. 13060906481 to Dr Gao) and Young Investigator Grant for diabetes study from Novo Nordisk (grant no. 2012 to Dr Gao).

\section{References}

1. Lee HB, Yu MR, Yang Y, Jiang Z and Ha H: Reactive oxygen species-regulated signaling pathways in diabetic nephropathy. J Am Soc Nephrol 14 (8 Suppl 3): S241-S245, 2003.

2. Nishikawa T and Araki E: Impact of mitochondrial ROS production in the pathogenesis of diabetes mellitus and its complications. Antioxid Redox Signal 9: 343-353, 2007.

3. Forbes JM, Coughlan MT and Cooper ME: Oxidative stress as a major culprit in kidney disease in diabetes. Diabetes 57: 1446-1454, 2008.

4. Giacco F and Brownlee M: Oxidative stress and diabetic complications. Circ Res 107: 1058-1070, 2010.

5. Sedeek M, Callera G, Montezano A, Gutsol A, Heitz F, Szyndralewiez C, Page P, Kennedy CR, Burns KD, Touyz RM and Hébert RL: Critical role of Nox4-based NADPH oxidase in glucose-induced oxidative stress in the kidney: Implications in type 2 diabetic nephropathy. Am J Physiol Renal Physiol 299: F1348-F1358, 2010.

6. Hancock JT, Desikan R and Neill SJ: Role of reactive oxygen species in cell signalling pathways. Biochem Soc Trans 29: 345-350, 2001.

7. Youn JY, Gao L and Cai H: The p47phox- and NADPH oxidase organiser 1 (NOXO1)-dependent activation of NADPH oxidase 1 (NOX1) mediates endothelial nitric oxide synthase (eNOS) uncoupling and endothelial dysfunction in a streptozotocin-induced murine model of diabetes. Diabetologia 55: 2069-2079, 2012.

8. Jha JC, Thallas-Bonke V, Banal C, Gray SP, Chow BS, Ramm G, Quaggin SE, Cooper ME, Schmidt HH and Jandeleit-Dahm KA: Podocyte-specific Nox4 deletion affords renoprotection in a mouse model of diabetic nephropathy. Diabetologia 59: 379-389, 2016.

9. Lee DY, Wauquier F, Eid AA, Roman LJ, Ghosh-Choudhury G, Khazim K, Block K and Gorin Y: Nox4 NADPH oxidase mediates peroxynitrite-dependent uncoupling of endothelial nitric-oxide synthase and fibronectin expression in response to angiotensin II: Role of mitochondrial reactive oxygen species. J Biol Chem 288: 28668-28686, 2013.

10. Gorin Y, Cavaglieri RC, Khazim K, Lee DY, Bruno F, Thakur S, Fanti P, Szyndralewiez C, Barnes JL, Block K and Abboud HE: Targeting NADPH oxidase with a novel dual Nox1/Nox4 inhibitor attenuates renal pathology in type 1 diabetes. Am J Physiol Renal Physiol 308: F1276-F1287, 2015.

11. Eid AA, Lee DY, Roman LJ, Khazim K and Gorin Y: Sestrin 2 and AMPK connect hyperglycemia to Nox4-dependent endothelial nitric oxide synthase uncoupling and matrix protein expression. Mol Cell Biol 33: 3439-3460, 2013.

12. Prabhakar S, Starnes J, Shi S, Lonis B and Tran R: Diabetic nephropathy is associated with oxidative stress and decreased renal nitric oxide production. J Am Soc Nephrol 18: 2945-2952, 2007.

13. Chen H, Zheng Z, Li R, Lu J, Bao Y, Ying X, Zeng R and Jia W: Urinary pigment epithelium-derived factor as a marker of diabetic nephropathy. Am J Nephrol 32: 47-56, 2010.

14. Wang JJ, Zhang SX, Mott R, Knapp RR, Cao W, Lau K and Ma JX: Salutary effect of pigment epithelium-derived factor in diabetic nephropathy: Evidence for antifibrogenic activities. Diabetes 55: 1678-1685, 2006.
15. Tombran-Tink J, Chader GG and Johnson LV: PEDF: A pigment epithelium-derived factor with potent neuronal differentiative activity. Exp Eye Res 53: 411-414, 1991.

16. Tombran-Tink J and Barnstable CJ: PEDF: A multifaceted neurotrophic factor. Nat Rev Neurosci 4: 628-636, 2003.

17. Becerra SP: Focus on molecules: Pigment epithelium-derived factor (PEDF). Exp Eye Res 82: 739-740, 2006.

18. Mao T, Gao L, Li H and Li J: Pigment epithelium-derived factor inhibits high glucose induced oxidative stress and fibrosis of cultured human glomerular mesangial cells. Saudi Med J 32: 769-777, 2011.

19. Gao L, Pung YF, Zhang J, Chen P, Wang T, Li M, Meza M, Toro $\mathrm{L}$ and Cai H: Sepiapterin reductase regulation of endothelial tetrahydrobiopterin and nitric oxide bioavailability. Am J Physiol Heart Circ Physiol 297: H331-H339, 2009.

20. Setsukinai K, Urano Y, Kakinuma K, Majima HJ and Nagano T: Development of novel fluorescence probes that can reliably detect reactive oxygen species and distinguish specific species. J Biol Chem 278: 3170-3175, 2003.

21. Sheikpranbabu S, Haribalaganesh R and Gurunathan S: Pigment epithelium-derived factor inhibits advanced glycation end-products-induced cytotoxicity in retinal pericytes. Diabetes Metab 37: 505-511, 2011.

22. Gao L, Huang W and Li J: NOX1 abet mesangial fibrogenesis via iNOS induction in diabetes. Mol Cell Biochem 382: 185-191, 2013.

23. Cheng $\mathrm{G}$ and Lambeth JD: NOXO1, regulation of lipid binding, localization, and activation of Nox1 by the Phox homology (PX) domain. J Biol Chem 279: 4737-4742, 2004.

24. Li X, Liu W, Wang Q, Liu P, Deng Y, Lan T, Zhang X, Qiu B, Ning $\mathrm{H}$ and Huang $\mathrm{H}$ : Emodin suppresses cell proliferation and fibronectin expression via p38MAPK pathway in rat mesangial cells cultured under high glucose. Mol Cell Endocrinol 307: $157-162,2009$.

25. Ho C, Lee PH, Huang WJ, Hsu YC, Lin CL and Wang JY: Methylglyoxal-induced fibronectin gene expression through Ras-mediated NADPH oxidase activation in renal mesangial cells. Nephrology (Carlton) 12: 348-356, 2007.

26. Liu CM, Qi XL, Yang YF and Zhang XD: Betulinic acid inhibits cell proliferation and fibronectin accumulation in rat glomerular mesangial cells cultured under high glucose condition. Biomed Pharmacother 80: 338-342, 2016.

27. Fang S, Jin Y, Zheng H, Yan J, Cui Y, Bi H, Jia H, Zhang H, Wang Y, Na L, et al: High glucose condition upregulated Txnip expression level in rat mesangial cells through ROS/MEK/MAPK pathway. Mol Cell Biochem 347: 175-182, 2011.

28. Yuan Z, Feng W, Hong J, Zheng Q, Shuai J and Ge Y: p38MAPK and ERK promote nitric oxide production in cultured human retinal pigmented epithelial cells induced by high concentration glucose. Nitric Oxide 20: 9-15, 2009.

29. Shatanawi A, Lemtalsi T, Yao L, Patel C, Caldwell RB and Caldwell RW: Angiotensin II limits NO production by upregulating arginase through a p38 MAPK-ATF-2 pathway. Eur J Pharmacol 746: 106-114, 2015.

30. Yang J, Duh EJ, Caldwell RB and Behzadian MA: Antipermeability function of PEDF involves blockade of the MAP kinase/GSK/beta-catenin signaling pathway and uPAR expression. Invest Ophthalmol Vis Sci 51: 3273-3280, 2010.

31. Gao S, Li C, Zhu Y, Wang Y, Sui A, Zhong Y, Xie B and Shen X: PEDF mediates pathological neovascularization by regulating macrophage recruitment and polarization in the mouse model of oxygen-induced retinopathy. Sci Rep 7: 42846, 2017.

32. Wen H, Liu M, Liu Z, Yang X, Liu X, Ni M, Dong M, Luan X, Yuan Y, Xu X and Lu H: PEDF improves atherosclerotic plaque stability by inhibiting macrophage inflammation response. Int $\mathrm{J}$ Cardiol 235: 37-41, 2017.

33. Pacher P and Szabo C: Role of the peroxynitrite-poly(ADP-ribose) polymerase pathway in human disease. Am J Pathol 173: 2-13, 2008.

34. Kar S and Kavdia M: Endothelial $\mathrm{NO}$ and $\mathrm{O}_{2}$.- production rates differentially regulate oxidative, nitroxidative, and nitrosative stress in the microcirculation. Free Radic Biol Med 63: 161-174, 2013.

35. Kouti L, Noroozian M, Akhondzadeh S, Abdollahi M, Javadi MR, Faramarzi MA, Mousavi S and Ghaeli P: Nitric oxide and peroxynitrite serum levels in Parkinson's disease: Correlation of oxidative stress and the severity of the disease. Eur Rev Med Pharmacol Sci 17: 964-970, 2013. 
36. Harrison DG, Chen W, Dikalov S and Li L: Regulation of endothelial cell tetrahydrobiopterin pathophysiological and therapeutic implications. Adv Pharmacol 60: 107-132, 2010.

37. Stern D, Yan SD, Yan SF and Schmidt AM: Receptor for advanced glycation endproducts: A multiligand receptor magnifying cell stress in diverse pathologic settings. Adv Drug Deliv Rev 54: 1615-1625, 2002.
38. Yamagishi S, Nakamura K, Matsui T, Noda Y and Imaizumi T: Receptor for advanced glycation end products (RAGE): A novel therapeutic target for diabetic vascular complication. Curr Pharm Des 14: 487-495, 2008.

39. Schröder K, Weissmann N and Brandes RP: Organizers and activators: Cytosolic Nox proteins impacting on vascular function. Free Radic Biol Med 109: 22-32, 2017. 